\title{
Vorwort des Gasteditors
}

\author{
Online publiziert am 13. November 2014
}

Die 8. Europäische Stranggießkonferenz (8th European Continuous Casting Conference) fand vom 23. bis 26. Juni 2014 in Graz statt. Die erfolgreichen Vorgängerkonferenzen in Florenz, Madrid, Düsseldorf, Birmingham, Nizza und Riccione legten die Latte hoch, doch die Grazer Altstadt und der Grazer Congress mit dem stilvollen Stefaniensaal als Veranstaltungszentrum konnten die Erwartungen mehr als erfüllen. Mehr als 650Teilnehmer fanden den Weg nach Graz, um sich in drei Tagen bei rund 140 Vorträgen mit Fachleuten aus aller Welt auszutauschen. Eine Ausstellung der bekanntesten Zulieferer und Anlagenbauer bildete einen attraktiven Rahmen.

Einen klaren fachlichen Schwerpunkt bildeten zahlreiche eingereichte Vorträge über den Einsatz der numerischen und physikalischen Simulation als Werkzeug der Stranggießforschung. So lag es nahe im Vorfeld der ECCC ein Symposium zum Themenschwerpunkt „Numerical and physical modelling" zu organisieren. Meinen Kollegen assoz. Prof. Menghuai Wu und assoz. Prof. Ernst Gamsjäger sei dafür ganz herzlich gedankt.

In der Eröffnungsveranstaltung wurden im Rahmen der "Manfred Wolf Memorial Lecture" ein großer Absolvent der Montanuniversität und seine Leistungen für die Stranggießforschung gewürdigt. Professor Wilfried Kurz, ebenfalls ein Absolvent der Montanuniversität, hielt diesen Vortrag und führte die Zuhörer an die faszinierende Welt der Erstarrungsforschung und deren Bedeutung für die Weiterentwicklung des Stranggießprozesses heran.

Neun ausgewählte internationale Experten aus dem industriellen und akademischen Umfeld konnten für "Key note lectures" gewonnen werden, um die zukünftigen Trends in Forschung und Entwicklung aufzuzeigen.

Ein weiterer Höhepunkt war die erstmalige Verleihung des „ECCC Young Scientist Award“: Acht junge Wissenschaftler wurden eingeladen, ihre Arbeiten vor großem Publikum zu präsentieren, und die Mitglieder des wissenschaftlichen Beirats wählten ihre Favoriten. Mit Dr. Jakob Six konnte ein Mitarbeiter der voestalpine Stahl den Bewerb für sich entscheiden und beim Gesellschafts- abend im Brauhaus Puntigam den mit 3000 Euro dotierten Preis, gesponsert von Siemens VAI Metals Technologies, entgegennehmen.

Mit einem Empfang durch die steiermärkische Landesregierung in der Aula der alten Universität und ausgebuchten Exkursionen zu voestalpine Stahl, voestalpine Stahl Donawitz und Acroni ging eine außergewöhnlich erfolgreiche Konferenz zu Ende.

Der große Erfolg der Veranstaltung ist auch ganz wesentlich dem Bemühen der ASMET (Yvonne Dworak und Dr. Bruno Hribernik) zu verdanken.

Für das vorliegende Heft durfte ich vier Konferenzbeiträge auswählen. Ich habe mich für Artikel aus Österreich entschieden, überwiegend Produkte von K1-MET und K2MPPE-Projekten, deren Qualität den hohen Stellenwert der Stranggießforschung in Österreich unterstreicht. Bei Alexander Mayerhofer bedanke ich mich für die wertvolle Unterstützung.

\section{Bernhard}

Leoben, Österreich

E-Mail: christian.bernhard@unileoben.ac.at 